\title{
Services innovants et action publique pour dynamiser les économies territoriales : éléments d'analyse
}

\section{Innovative services and public action to boost territorial economies:} elements of analysis

\author{
Michelle Mongo ${ }^{1}$ \\ ${ }^{1}$ Mines Saint-Etienne, Institut Henri Fayol, Coactis (EA 4161), 158, cours Fauriel, F-42023 Saint-Etienne, France, \\ michelle.mongo@emse.fr
}

RÉSUMÉ. Dans un contexte économique ou l'innovation, conditionne de plus en plus les perspectives de croissance économique des territoires et/ou le secteur des services représente près de $70 \%$ du PIB des pays développés. La problématique de l'innovation dans le secteur des services constitue un enjeu majeur pour le développement économique des territoires. Or, la question de l'innovation dans les services bénéficie que de peu d'évidences empiriques et il existe à ce jour un nombre limité de travaux permettant de caractériser les processus d'innovation dans ce secteur ainsi que ces implications en matière de politiques publiques sur les territoires. Or cela paraît paradoxal compte tenu de la part importante que représente le secteur des services dans nos économies. L'objectif de cette contribution est de proposer une analyse permettant de mieux comprendre les enjeux relatifs à l'innovation dans le secteur des services. Ainsi, il s'agira de revenir sur la définition de l'innovation dans les services, les spécificités des mécanismes à l'œuvre dans ce cadre, les impacts territoriaux observés et les politiques publiques engagées et permettant de favoriser les dynamiques locales d'innovation de ces activités.

ABSTRACT. The current economic context is one in which innovation increasingly conditions the economic growth prospects of territories and where the service sector represents almost $70 \%$ of the GDP of developed countries. Innovation in the service sector is a major issue for the economic development of territories. However, there is little empirical evidence on the question of service innovation and there is, to date, a limited number of studies allowing us to characterize the innovation processes in this sector, as well as their implications in terms of public policies. However, this seems paradoxical, given the significant share that the service sector represents in our economies. The objective of this article is to provide an analysis that will help us to better understand the challenges relating to innovation in the service sector. First, the definition of service innovation will be presented. Secondly, the specifics of the innovation processes in this sector will be presented, then the territorial impacts observed and, finally, the public policies that have been implemented to promote the local dynamics of innovation in these activities.

MOTS-CLÉS. Innovation dans les services, Territoires, Services à Forte Intensité de Connaissances (SFIC), Politique d'innovation.

KEYWORDS. Service innovation, Territories, Knowledge Intensive Business Services (KIBS), Innovation policy.

\section{Introduction}

Les économies contemporaines sont qualifiées aujourd'hui de sociétés de service. En effet, le secteur des services emploie les $2 / 3$ de la population des pays développés et sa part dans leur PIB est d'environ 72\% (Banque mondiale, base de données 2020). En France par exemple, les services emploient en 2018 près de $76 \%$ de la population active contre $14 \%$ dans l'industrie, $7 \%$ dans la construction et 3\% dans l'agriculture [INS 18]. Au sein du secteur des services, les activités axées sur les connaissances intensives en savoir semblent prendre une importance croissante. Plus précisément, les services intensifs en savoir représentent en 2019 près de 39\% de l'emploi total des pays de l'Union Européenne (soit une hausse de près de 3,5 points entre 2008 et 2019) (Eurostat, base de données). Cette particularité traduit en partie l'évolution des économies vers une nouvelle phase de développement. Cette phase est basée sur la connaissance et succède selon les spécialistes à une phase d'accumulation de capital physique. On parle dès lors "d'économie de la connaissance " pour caractériser la dynamique des économies actuelles. L'économie de la connaissance porte l'attention sur des éléments intangibles relatifs à la production de savoirs, de sciences, de compétences techniques et 
aussi de « capital humain ». Face à ces nouveaux enjeux, c'est toute la question de l'innovation ${ }^{1}$ qu'il faut considérer, car cette dernière conditionne de plus en plus les perspectives de croissance économique des territoires.

La littérature semble confirmer que le positionnement technologique reste de loin un élément important du niveau de croissance des territoires avec pour principal déterminant le capital humain [BAR 00] [LUC 88] [ROM 90]. Plus précisément, en s'appuyant sur la notion d'externalités locales de connaissances sources de rendements croissants, l'analyse économique des relations entre innovation et territoire a connu un développement important dans les vingt dernières années. Elle place clairement l'innovation associant positionnement technologique et capacité créative, comme un des éléments essentiels en matière de développement territorial.

En revanche, les relations entre Innovation, Services et Territoires restent encore ambigües et beaucoup moins étudiées. Au sein du secteur des services, la problématique de l'innovation a été jusqu'à présent peu analysée. Au regard des études portant sur l'innovation depuis ces deux dernières décennies, on constate que les analyses se sont essentiellement intéressées aux innovations technologiques opérant au sein du secteur industriel. Or cela paraît paradoxal compte tenu de la part importante que représente le secteur des services dans nos économies.

Une des explications possibles à ce manque d'études sur l'innovation dans ce secteur est que :

" les services n'innovent pas ou se contentent d'adopter des innovations technologiques produites dans l'industrie » [GAL 96: 2]

Cette représentation de la structure économique centrée sur l'industrie et la production matérielle relègue les services et les fonctions de services à la périphérie [GAL 96]. La forte contribution des services supérieurs à l'innovation des entreprises industrielles [MUL 01] [WER 18] renforce néanmoins les questionnements concernant le rôle de ces activités dans les dynamiques territoriales d'innovation.

Toutefois, les différentes études réalisées sur la thématique des services et/ou la géographie de l'innovation [CHA 12] soulignent le travail encore à mener pour aboutir à une meilleure perception des mécanismes à l'œuvre lorsqu'on parle d'innovation dans les services, et que l'on souhaite définir des indicateurs permettant de mesurer ces phénomènes et leurs impacts sur les territoires. Ces différents travaux, constituent autant d'éléments qui justifient la réalisation de cette contribution. L'objectif de cette contribution est de proposer une analyse permettant de mieux comprendre les enjeux relatifs à l'innovation dans le secteur des services. Ainsi, il s'agira de revenir sur la définition de l'innovation dans les services, les spécificités des mécanismes à l'œuvre dans ce cadre, les impacts territoriaux observés et les politiques publiques engagées et permettant de favoriser les dynamiques locales d'innovation de ces activités.

\section{Innovation dans les services : de quoi parle-t-on ?}

\subsection{Qu'est-ce qu'un service}

La particularité du secteur des services est qu'il englobe l'ensemble des activités qui ne sont pas directement associées à la production de biens et aux activités du secteur primaire [BEN 97] [NOH 92]

\footnotetext{
${ }^{1}$ D'après le Manuel d'Oslo (2005), une innovation se définit comme : l'introduction sur le marché d'un produit (bien ou service) nouveau ou significativement modifié par rapport aux produits (biens ou services) précédemment élaborés par l'entreprise. La nouveauté ou l'amélioration se mesurent par rapport aux caractéristiques essentielles du produit (bien ou service), de ses spécifications techniques ou de la convivialité ; ou l'introduction dans l'entreprise de procédés de production, de méthodes concernant la fourniture de services ou la livraison de produits, d'activité de supports nouveaux ou significativement modifiés ; ou l'introduction de solutions nouvelles ou nettement améliorées concernant l'organisation ou le marketing.
} 
[OEC 00]. Selon l'Insee, les services recouvrent un champ large d'activités allant du commerce à l'administration, en passant par les transports, l'immobilier, les activités financières, les services aux entreprises, l'éducation, la santé et l'action sociale. En ce sens, il existe un nombre important de définitions relatives au secteur des services. Pour Hill [Hil 77] par exemple, le service diffère du bien dans la mesure où il n'est pas appropriable. En effet, si les biens et les services présentent une caractéristique commune à savoir d'être échangeables et de pouvoir faire l'objet d'une transaction entre plusieurs agents, ils diffèrent en ce qui est échangé. Le bien échangé est un produit appropriable, il n'en est pas de même du service.

Pour Houard et Belleflamme [HOU 87] échanger un service consiste à opérer un changement dans la condition d'une personne ou d'un bien, ce changement nécessitant l'intervention d'un autre agent. Enfin, à la différence du bien, lorsqu'un consommateur paye un service, il paye avant tout la conception et l'organisation d'une séquence d'action [CAL 97].

Cook, Goh et Chung [COO 09] reconnaissent qu'il n'existe pas de définition du service qui soit capable de tenir compte des particularités propres à chaque activités de service.

De son côté, Gadrey [GAD 92] [GAD 03] admet que le service fait intervenir une relation complexe entre trois pôles : le prestataire, le client (qui peut intervenir dans la réalisation du service ou dans le contrôle du service) et la réalité, modifiée ou travaillée par le prestataire au bénéfice du client et consistant soit en biens, en systèmes techniques, en informations, en individus ou encore en organisation. Pour Gadrey, Gallouj et Weinstein produire un service consiste :

"to organize a solution to a problem which does not principally involve supplying a good. It is to place a bundle of capabilities and competencies (human, technological, organizational) at the disposal of a client and to organize a solution, which may be given to varying degrees of precision." [GAD 95]

Cette définition fait consensus dans la littérature sur les services, elle est la plus appropriée dans la mesure où elle fait intervenir les quatre principales caractéristiques du service [JON 03] [MIL 06] [JBDM03, Mile06] à savoir :

\section{L'intangibilité}

L'activité de service revêt un caractère immatériel relativement important. C'est ce qui la différencie des produits manufacturés. En effet, le bien est caractérisé par un objet tandis que le service constitue une action, un effort, de fait intangible. Néanmoins au sein des services, la notion d'intangibilité varie d'une activité à une autre. On parle de degré de tangibilité pour distinguer les " services purs » (tels que les services de conseil) pour lesquels le degré de tangibilité est quasi nul, des services plus tangibles dont la prestation est difficilement dissociable des produits qui l'accompagnent (services de transports ou encore restauration rapide).

\section{La périssabilité}

Les services sont difficilement stockables compte tenu de leur caractère intangible. Ils sont, de fait, éphémères, car la production et la consommation du service interviennent en général de façon simultanée.

\section{L'hétérogénéité}

Le service est souvent adapté aux besoins des clients, ce qui le rend plus hétérogène en comparaison des biens standardisés.

4. La réciprocité 
En comparaison du bien, le service est souvent le résultat d'une coproduction entre un prestataire et son client ou un client et son fournisseur, ce qui aboutit le plus souvent à un produit-service personnalisé.

\subsection{Que signifie innover dans les services?}

La notion d'innovation dans les services est assez complexe puisqu'elle suppose des caractéristiques d'innovation différentes de celles du secteur industriel. Dans l'industrie, l'objet de l'innovation se matérialise habituellement par un produit (bien) tandis que dans les services l'objet de l'innovation est assez flou, car la distinction entre produit et procédé est peu évidente [DJE 00]. De même, le degré de nouveauté dans l'industrie se matérialise en majorité par une dimension radicale en comparaison avec le secteur des services dont les innovations sont plutôt de nature incrémentale.

Du côté de la littérature théorique, la perception de l'innovation dans le secteur des services a évolué selon trois principaux courants d'analyse reconnaissant progressivement l'existence de dynamique d'innovation au sein de ce secteur [GAL 09]. Parmi ces courants, on distingue l'approche : Technologiste ; Servicielle et Intégratrice.

L'approche technologiste a analysé l'innovation dans les services strictement à travers un cadre conceptuel et des indicateurs propres au secteur industriel. Ce cadre suppose ainsi que les entreprises de service sont de simples utilisateurs des technologies produites au sein de l'industrie et qu'elles sont par conséquent peu innovantes. Un exemple de typologie des innovations basée sur l'approche technologiste est celle proposée par Soete et Miozzo [SOE 89] et qui découle des travaux de Pavitt [PAV 84]. Ces auteurs distinguent trois types de firmes ou de secteurs et admettent que les schémas d'innovation ont lieu dans ces firmes à différentes échelles :

1. Les firmes «dominées par les fournisseurs » d'équipements et de systèmes techniques : Ce sont principalement des firmes qui participent peu à la production des technologies de process qu'elles utilisent, on y distingue les services aux particuliers (blanchisserie, restauration) et les services publics et sociaux (éducation, santé, administration)

2. Les firmes en réseaux : Ce sont des firmes dont la trajectoire technologique repose sur la baisse des coûts et la stratégie de mise en réseau. L'innovation repose ici sur la standardisation (il s'agit par exemple de services à dominante de traitement d'informations codifiées : finance, assurance, communication).

3. Les fournisseurs spécialisés et les services fondés sur la science : Ces firmes sont de grands producteurs d'innovations technologiques car elles sont en général très engagées dans des activités de recherche et développement et de conception de logiciel (cette catégorie comprend les grands réseaux internationaux d'audit et de conseil et aussi les grandes sociétés de services et d'ingénierie informatique).

L'approche servicielle, portée par l'université de Lille à partir des années 90 souligne les particularités du produit/process au sein des services et démontre la nature complexe des processus d'innovation au sein de ce secteur. Ces travaux reconnaissent que l'interactivité de l'activité de service encourage la mise en œuvre de forme particulière d'innovation basée sur les relations clients/fournisseurs. Ces différentes innovations, sont largement perçues comme étant de nature non technologique. Elles émergent le plus souvent de services purs pour lesquels les critères d'immatérialité et de coproduction de l'output sont les plus marqués. Gallouj et Gallouj [GAL 96] par exemple, analysent l'innovation au sein des services de conseils et relèvent trois types d'innovation :

1. L'innovation - valorisation ou l'innovation had hoc 
L'innovation had hoc consiste en l'utilisation de connaissances acquises au cours d'expériences passées afin de créer des connaissances nouvelles. Cette forme d'innovation est produite en étroite collaboration avec le client. Il s'agit par exemple de certains montages juridiques et fiscaux favorables et à destination des clients.

\section{L'innovation - anticipation}

L'innovation - anticipation consiste à détecter un besoin nouveau (en valeur absolue ou relative) et d'élaborer par collecte et analyse un capital de connaissances et d'expertises (par exemple l'investissement dans les domaines de droit de l'environnement ou droit de l'informatique pour certains cabinets de conseils).

\section{L'innovation - objectivation}

L'innovation - objectivation est une catégorie assez hétérogène d'innovation, elle veut donner une certaine forme de matérialité au service. Elle fait appel à plusieurs formes d'innovation qui peuvent relever de l'innovation de marketing, d'innovation organisationnelle, etc.

Enfin L'approche intégratrice est considérée comme la plus prometteuse en termes d'avancée théorique. Elle repose sur le constat d'une réduction des frontières entre les biens et les services et sur le fait qu'un certain nombre de services a tendance à s' « industrialiser» tandis que la production de certains biens se «tertiarise ». Les biens et services évoluent vers un continuum biens/services dans lequel, le produit (biens/services) est redéfini en termes de fonctions ou encore de caractéristiques. Un exemple de typologie selon l'approche intégratrice est celle fournie par Gallouj et Weinstein [GAL 97]. En redéfinissant la nature du produit en termes de caractéristiques, les auteurs identifient cinq formes d'innovation au sein du secteur des services. Parmi lesquelles on retrouve l'innovation:

1. Radicale : Il s'agit de la création d'un produit/service totalement nouveau.

2. D'amélioration : Ce type d'innovation améliore un certain nombre de caractéristiques d'un produit/service sans modifier la structure du système.

3. Incrémentale (ou de perfectionnement) : Consiste à ajouter des éléments nouveaux qui améliorent la performance du produit service.

4. Ad hoc : Il s'agit d'une innovation qui solutionne le problème d'un client et qui se fait en étroite collaboration avec ce dernier.

5. De recombinaison : C'est une innovation par ajout de caractéristiques

Au final ces différentes classifications issues de la littérature théorique montrent l'extrême diversité des modes d'innovation dans les services. Les typologies fondées sur les approches technologistes et servicielles présentent la limite de n'analyser qu'une partie des modes d'innovation dans les services (technologique ou non technologique). L'approche intégratrice, bien qu'elle présente l'intérêt d'analyser les deux types d'innovation développés au sein des entreprises de services, elle se focalise essentiellement sur la nature des innovations et évoque assez peu le rôle des différents acteurs (le client, le fournisseur et la firme de service) dans le processus d'innovation.

D'un point de vue empirique, l'intérêt pour le secteur des services dans les études économétriques et en particulier celles qui tentent d'éclairer les processus d'innovation [MAI 10] restent encore limitées et largement focalisées sur les innovations technologiques principalement présentes au sein du secteur industriel. Néanmoins, on récence un certain nombre d'études empiriques qui tentent d'expliquer quantitativement les différences dans les processus d'innovation entre les services et l'industrie [ARU 07]. Ces dernières soulignent que les spécificités en matière d'innovation dans les services résident dans un faible investissement dans des activités de R\&D, l'intérêt pour d'autres formes d'innovation axées notamment sur le changement organisationnel, l'investissement plus intense dans la formation du 
personnel, les relations étroites entretenues avec les clients de services et les fournisseurs de technologie, et enfin l'existence de différences intra-sectorielles liées en partie à la nature des innovations développées.

A partir de l'exploitation de l'enquête CIS2008², Mongo [MON 13] affine les résultats de ces travaux en montrant dans quelle mesure les différences en matière d'innovation au sein du secteur des services et de l'industrie résident dans les formes d'innovations développées. En outre, l'innovation technologique dont le développement est plus fortement déterminé par un engagement poussé dans des activités de R\&D semble bénéficier davantage aux entreprises industrielles et/ou à forte intensité technologique (industrie de haute et moyenne haute technologie et services technologiques). À l'inverse l'innovation non technologique particulièrement présente au sein des entreprises de services caractérisées par un niveau technologique faible (services intellectuels) apparaît fortement déterminée par la formation du personnel, les acquisitions de machines, des partenariats client/fournisseurs privilégiés et un niveau local des coopérations.

Ces résultats empiriques soulignent les dynamiques d'innovation à l'œuvre au sein du secteur des services et mettent également en lumière l'existence de différences intra-sectorielles en matière d'innovation (les services d'accueil et d'appui apparaissent ainsi comme les activités de services les moins innovantes). Ces travaux justifient par ailleurs l'arbitrage opéré dans la littérature concernant le type d'activités de services à prendre en compte pour l'analyse empirique des dynamiques d'innovation dans les services et de leurs effets sur les territoires. Ces activités de services sont présentées dans la suite de cette section.

\subsection{Quels services pour l'analyse de l'innovation?}

Le périmètre vaste des activités contenues dans le secteur des services a contraint les spécialistes à opérer un arbitrage sur le type d'activités à prendre en compte pour l'analyse et la mesure de l'innovation dans les services. Au regard des résultats de la littérature [HER 00] [MIL 95] les Services à Forte Intensité de Connaissances ${ }^{3}$ (SFIC) apparaissent comme les plus appropriés pour l'analyse des phénomènes d'innovation dans les services.

Selon l'OCDE, les Services à Forte Intensité de Connaissances (SFIC) désignent :

"Les activités de service produites ou intégrées par des entreprises ou par des acteurs du secteur public, dans le cadre d'activités manufacturières ou de services, parallèlement à la production manufacturière, ou en tant que services autonomes. La plupart des organisations du secteur public et des entreprises ont recours à des services à forte intensité de savoirs dans leur fonctionnement quotidien, services qu'elles se procurent soit en interne, soit auprès de fournisseurs externes appartenant aux secteurs privés ou publics». [OEC 06:7]

Les SFIC intègrent des activités de « services technologiques » ainsi que des activités de "services intellectuels » [Cord11] [MIL 95].

\footnotetext{
${ }^{2}$ Enquête communautaire sur l'innovation

${ }^{3}$ L'abréviation KIBS ou Knowledge Intensive Business Services est habituellement utilisée dans la littérature en langue anglaise pour désigner les SFIC voir [MKFB95].
} 


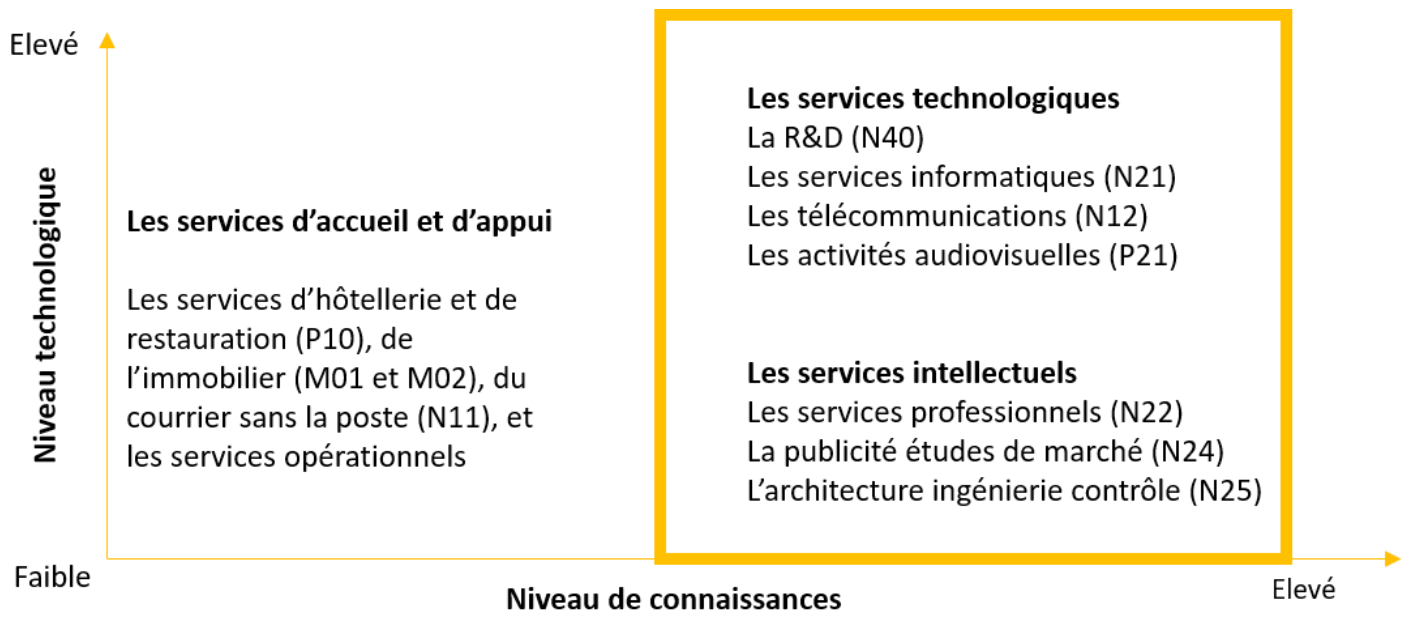

Figure 1. Source auteur sur la base de [Cord11]

La différence entre ces activités de services dits «innovants » et les autres repose sur le degré de connaissances incorporé au sein même de ces activités [MUL 09]. En effet, l'une des caractéristiques majeures des SFIC réside dans le fait que leurs prestations reposent généralement sur une expertise forte dans un domaine précis, la main d'œuvre y est fortement qualifiée. Miles et al [MIL 95] identifient trois caractéristiques principales communes aux SFIC :

1. Ils s'appuient fortement sur les connaissances professionnelles.

2. Ils sont eux-mêmes des sources d'information et de connaissances. Ils utilisent ces connaissances pour produire des services auprès de leurs clients.

3. Ils fournissent des services principalement aux entreprises et sont très compétitifs.

\section{Géographie de l'innovation dans les Services à Forte Intensité de Connaissances et impacts territoriaux}

\subsection{Les faits stylisés de la géographie des Services à Forte Intensité de Connaissances}

Les enjeux de localisation des SFIC sont importants au regard des effets d'entrainement en matière de dynamique territoriale d'innovation qu'elles engendrent [MUL 01].

Toutes les études observant la distribution spatiale des activités d'innovation constatent que les activités innovantes sont plus concentrées que les activités de production. En effet, les faits stylisés montrent que la plupart des activités d'innovation se concentrent dans quelques pays, quelques régions, voire quelques zones urbaines de ces régions [CHA 12]. Concernant les SFIC, les effets de concentration en Europe sont plus renforcés comparativement aux activités industrielles. Les plus fortes concentrations de SFIC se trouvent dans les grandes régions métropolitaines. Au sein de ces dernières, des disparités interrégionales sont perceptibles et se caractérisent par des différences en matière de spécialisation sectorielles dans les branches de SFIC [SIM 06]. Au Royaume-Uni, par exemple, Newcastle présente la plus forte concentration dans les services techniques, suivie de Manchester. De même en Allemagne, Hambourg se caractérise par une spécialisation dans le secteur de la publicité tandis que Munich est spécialisé dans les services informatiques et les services de recherche et développement.

Du côté de la littérature empirique, la concentration des activités innovantes est habituellement expliquée par la dimension géographique des externalités de connaissances qui s'effectue sur une base locale lorsque les connaissances sont de nature tacite. Ces dernières circulent à travers différents canaux issus notamment des interactions inter-entreprises, de la recherche privé-publique, de la 
concentration d'une main d'œuvre qualifiée et de la forte mobilité inter-firmes, de la nature des structures locales (diversifiées ou spécialisées) et enfin du caractère résiliaire de la production de connaissances [CHA 12]. Certains de ces facteurs contribuent largement à renforcer les effets de localisation des SFIC compte tenu des mécanismes d'innovation à l'œuvre au sein de ce secteur. McCann en étudiant le lien entre les interactions de face à face, le développement de l'innovation et le comportement de localisation des firmes, démontre que la localisation des firmes innovantes est dépendante de l'intensité des contacts de face à face :

"(...) different types of innovative activities will be spatially distributed in different locations, according to the relative importance which face-to-face contact plays in the firm's innovation processes." [MCC 07: 132]

Plus précisément, les firmes sont d'autant plus concentrées dans l'espace que leurs activités d'innovation nécessitent des contacts de face à face intenses et inversement. Les conclusions portées par l'étude de McCann [MCC 07] sont intéressantes notamment pour comprendre la localisation des SFIC. En effet, elles supposent que pour le cas des SFIC, les effets de concentration peuvent être plus renforcés dans la mesure où l'activité d'innovation au sein de ces secteurs nécessite des interactions intenses entre les clients de services et les fournisseurs de technologie. Ces derniers contribuent par ailleurs aux dynamiques territoriales d'innovation.

\subsection{La contribution des Services à Forte Intensité de Connaissances aux dynamiques territoriales d'innovation}

La contribution des SFIC aux dynamiques territoriales d'innovation réside à la fois dans les effets directs (liés à leurs propres activités d'innovation) et indirects (liés à la contribution de l'innovation des entreprises industrielles) causés par leurs activités d'innovation [WER 18]. Hertog [HER 00] estime à ce propos que les SFIC peuvent être considérés comme :

- Des vecteurs d'innovations : principalement lorsqu'ils contribuent à transférer des connaissances existantes entre organisations, industries, réseaux, ou à l'intérieur de ceux-ci, de façon à les appliquer dans un nouveau contexte (activité de conseils ou de formation par exemple).

- Des facilitateurs d'innovations : principalement lorsqu'ils aident une organisation dans son processus d'innovation (service de technologie).

- Sources d'innovations : lorsqu'ils interviennent dans le lancement et le développement d'activités d'innovation au sein d'organisations clientes (R\&D, design).

Werner et Strambach [WERN 18] admettent que les activités des SFIC contribuent à accroitre la capacité d'adaptation des économies urbaines ce qui tend par ailleurs à renforcer les dynamiques locales d'innovation. Larsen [LAR 00] a montré qu'au Danemark les SFIC sont davantage orientés vers des activités d'innovation par rapport aux autres activités de services. Muller et Zenker [MUL 01] ont analysé le rôle des interactions dans l'innovation des PME industrielles et des SFIC au sein de régions françaises et allemandes. Les résultats de l'étude montrent que les entreprises industrielles qui interagissent avec les SFIC présentent un potentiel en matière d'innovation plus important comparativement à celles qui n'interagissent pas. Tether et Hipp [TET 02] ont analysé, à partir de données allemandes les schémas d'innovation et de compétitivité que remplissent les entreprises de services allemandes. Ils en concluent que les SFIC dépensent davantage en innovation (notamment dans la gestion de la main d'œuvre) que les services à faible intensité de savoir. Pour ces économistes, les SFIC sont plus susceptibles d'identifier des sources externes d'information et de s'engager dans la coopération que les autres activités de services. 
Les exemples d'études empiriques qui confirment les effets d'entrainement des SFIC en matière d'innovation sont nombreux ${ }^{4}$ et malgré cela la politique d'innovation (plus spécifiquement la politique d'innovation française) reste peu adaptée à ce secteur.

\section{Politique publique d'innovation : actions historiques et perspectives futures}

La politique d'innovation en Europe est orientée depuis un certain nombre d'années vers la promotion de la compétitivité des territoires. Les actions menées se traduisent par la mise en œuvre de programmes cadres de recherche et d'innovation (PCRD) visant à promouvoir la R\&D des acteurs de la recherche européenne par le biais d'un mécanisme d'appel à projet centré sur la R\&D. Elle est en ce sens clairement tournée vers le développement des innovations technologiques dans la mesure ou la R\&D constitue un des principaux facteurs de développement de l'innovation technologique [MAI 10]. En France, le dispositif le plus important en matière d'aide publique à la $R \& D$ privée est le crédit impôt recherche (CIR). Or ce dernier fait l'objet de vive critique du fait des effets de leviers peu significatifs observés et imputables à deux principaux facteurs : le manque de sélectivité et le fait qu'il ne représente qu'un input de l'innovation. En effet, le CIR ne cible pas formellement les secteurs d'activités qui doivent en bénéficier. Or la spécificité des SFIC et le développement des formes d'innovations non technologiques doivent faire l'objet de politique publique appropriée et non calquée sur celle de la R\&D. En effet, il est inopportun de calquer les mesures d'intervention publique en faveur de l'innovation élaborée pour le cas d'entreprises industrielles aux cas d'entreprises de services notamment à faible niveau technologique. D'autres spécialistes reconnaissent l'importance d'un ciblage vers des domaines à intensités technologiques fortes plus susceptibles de développer les innovations technologiques [LAL 11]. Duguet reconnaît d'ailleurs que l'une des principales limites des dispositifs fiscaux en matière de $R \& D$ est le risque d'effet d'aubaine :

"Par rapport aux subventions (...) les mesures fiscales ne font pas l'objet d'un examen préalable des projets de recherche et posent de ce fait avec plus d'acuité le problème de l'effet d'aubaine ». [DUG 08: p.5]

Par ailleurs, les évolutions sociétales (économie de l'usage, place du numérique, économie collaborative) montrent dans quelle mesure l'innovation ne découle pas uniquement de la R\&D. Elle est tirée par les usages, passe par le design, les modèles d'affaires et est en partie incrémentale.

En ce sens, la politique d'innovation européenne doit s'adapter face à ces évolutions et prendre en compte les grands défis économiques, sociétaux et environnementaux (économie verte et impact du changement climatique). C'est d'ailleurs dans ce cadre, que s'inscrit les orientations de la nouvelle politique européenne qui vise notamment à lutter contre les effets du réchauffement climatique [COM 17]. En France par exemple, Encoua [ENC 17] admet qu'au regard de la révolution numérique de la société française et de ses effets sur les usages, il est primordial que l'Etat repense la politique d'innovation au travers la mise en place de dispositifs d'accompagnement aux transformations sociétales induites. Cela se traduirait entre autres par le soutien envers les collaborations accrues entre les développeurs des technologies numériques et les usagers. Ainsi, au-delà des effets attendus en matière de création d'emplois, cette nouvelle politique d'innovation contribuerait à intégrer les usagers au cœur du processus d'innovation des entreprises.

\section{Conclusion}

Le secteur des services emploie les $2 / 3$ de la population des pays développés et sa part dans leur PIB est d'environ $70 \%$. Malgré cela, la question de l'innovation dans les services bénéficie que de peu d'évidences empiriques et il existe à ce jour un nombre limité de travaux nous permettant de

\footnotetext{
${ }^{4}$ Pour une revue de ces études le lecteur est invité à lire [MuZe01]
} 
caractériser les processus d'innovation dans ce secteur ainsi que leurs implications en matière de politiques publiques sur les territoires. Ce fait est lié aux caractéristiques propres des activités de services et aux modes d'innovation inhérents à ce secteur.

De ce point de vue, les travaux théoriques portant sur « l'économie des services » et «l'innovation dans les services » montrent dans quelle mesure la diversité des activités au sein du secteur des services, les définitions hétérogènes de l'activité de service, les difficultés d'application de la distinction entre innovations de produit et innovation de procédé, les formes d'innovations caractérisées par une dimension non technologique, etc. constituent autant d'éléments qui rendent difficile l'appréhension et la mesure de l'innovation dans les services. Ces différents éléments ont contraint les spécialistes à opérer un arbitrage sur le type d'activités à prendre en compte pour l'analyse et la mesure de l'innovation dans les services. Les Services à Forte Intensité de Connaissances (SFIC) apparaissent ainsi comme les activités les plus appropriés pour l'analyse des phénomènes d'innovation dans les services. Leurs processus d'innovation axés davantage sur la relation client/fournisseur et les interactions intersectorielles contribuent à les identifier comme des vecteurs, des facilitateurs et des sources d'innovation territoriales.

Mais les politiques publiques d'innovation restent encore trop largement centrées sur la promotion des activités de $R \& D$ opérées au sein de l'industrie. Or ces dernières sont peu présentes au sein des services à faibles intensités technologiques. En somme pour dynamiser l'innovation dans les services il est primordial de repenser la politique d'innovation en considérant la transformation progressive de nos sociétés, d'une économie de la propriété vers une économie des services et de l'usage. C'est d'ailleurs dans ce cadre que s'inscrit les orientations de la nouvelle politique européenne qui vient à la suite du programme européen Horizon 2020 (H2020).

\section{Bibliographie}

[AKC 07] ARUndel A., KANERVA M., VAN CRUYSEN A., Hollanders H. J. G. M., Innovation statistics for the European service sector. Brussels, 2007.

[BAR 00] BARRO R., Les facteurs de la croissance économique, Nouvelle édition, ECONOMICA 2000.

[BEN 97] BENSAHEL L., Introduction à l'économie du service. Presses Universitaire, 1997.

[CAL 97] CALLON M., LARÉDO P., RABEHARISOA V., Que signifie innover dans les services ? une triple rupture avec le modèle de l'innovation industrielle. In: La Recherche (1997), S. 34-36.

[CHA 12] ChAlaye S., MASSARD N., Géographie de l'innovation en Europe. Observer la diversité des régions françaises. Datar, 2012.

[COO 09] CoOK D.-P., GoH C.-H., CHung C.-H., "Service Typologies: a State of the Art Survey". In: Production and Operations Management Bd. 8 (2009), Nr. 3, S. 318-338.

[COM 17] COMMISSION EuROPEAN., Open Innovation Open Science Open to the World, 2017.

[COR 11] CORDELLIER C., Innovations et performances des sociétés :comparaison entre les services et l'industrie manufacturière, chapter Innovation et performances économiques, 2011.

[DJE 00] DJEllal F., Gallouj F., Le «casse-tête» de la mesure de l'innovation dans les services: enquête sur les enquêtes. In: Revue d'économie industrielle Bd. 93 (2000), Nr. 1, S. 7-28.

[DUG 08] DuGUET E., L'effet du crédit dimpôt-recherche sur le financement privé de la recherche. Technical report, Ministère de l'Enseignement Supérieur et de la Recherche (MESR), 2008.

[ENC 17] ENCAOUA D., Repenser les politiques d'innovation en France ? In: Revue française d'économie Bd. XXXII (2017), Nr. 3, S. 90.

[GAD 03] GADREY J., Socio-économie des services. Edition, Repères - La Découverte, 2003.

[GAD 92] GADREY J., L'économie des services. Edition, Repères - La Découverte, 1992.

[GAD 95] Gadrey J., Gallouj F., Weinstein O., New modes of innovation how services benefit industry. In: International Journal of Service Industry Management Bd. 3 (1995), S. 4-16. 
[GAL 96] Gallouj C., Gallouj F., L'innovation dans les services. Economica. Paris, 1996.

[GAL 09] Gallouj F., SAvona M., Innovation in services: A review of the debate and a research agenda. In: Journal of Evolutionary Economics Bd. 19 (2009), Nr. 2, S. 149-172.

[GAL 97] GALLOUJ F., WeInSTEIN O., Innovation in services. In: Research Policy Bd. 26 (1997), Nr. 4-5, S. 537-556ISBN 0048-7333.

[HER 00] HeRTOG P.-D., Knowledge-Intensive Business Services As Co-Producers Of Innovation. In: International Journal of Innovation Management Bd. 4 (2000), Nr. 4, S. 491-528.

[HIL 77] HILL, P., On goods and services. In: The review of income and Wealth (1977), S. 315-338.

[HOU 87] Houard J., Belleflamme C., Perspective du système productif : analyse des activités de services rendus aux entreprises en belgique, 1987.

[INS 18] INSEE: Emploi par région, 2018.

[JON 03] JONG JEROEN P.J., BRUINS A., DolfSMA W., MEIJAARD J., Innovation in service firms explored: what, how and why? Bd. 1, 2003.

[LAL 11] LALLEMENT R., L'aide publique aux entreprises en matière de rd et d'innovation: quelle efficacité ? In: Technical Report Bd. 01, 2011.

[LAR 00] LARSEN, J. N., Supplier-User Interaction in Knowledge-Intensive Business Services: Types of Expertise and Modes of Organization. In: Services and the Knowledge Based Economy. Mark Boden, 2000.

[LUC 88] LUCAS, R.E., On the Mechanics of Economic Development. In: Journal of Monetary Economics Bd. 22 (1988), Nr. 1, S. 3-42.

[MAI 10] MAIRESSE J., MOHNEN, P., Using innovation surveys for econometric analysis. Bd. 23. Maastricht, 2010.

[MCA 07] MCCANN, P.: Sketching out a model of innovation, face-to-face interaction and economic geography. In: Spatial Economic Analysis Bd. 2 (2007), S. 117-134.

[MIL 06] MILES I., Innovation in Services. In: MOWERY, J. F. AND D. C. (Hrsg.): The Oxford Handbook of Innovation, 2006.

[MIL 95] Miles I., Kastrinos N., Flanagan K., Bilderbeek R., Hertog P. D., Knowledge intensive business services : users, carriers and sources of innovation., 1995.

[MON 13] MONGO M., Les déterminants de l'innovation: Une analyse comparative service/ industrie à partir des formes d'innovation développées. In: Revue d'Economie Industrielle Bd. 143 (2013), Nr. 3, S. 71-108.

[MUL 09] Muller E., DOlOREuX D., What we should know about knowledge-intensive business services. In: Technology in Society Bd. 31, Elsevier Ltd (2009), Nr. 1, S. 64-72.

[MUZ 01] MULLER E., ZENKER A., Business services as actors of knowledge transformation and diffusion: some empirical findings of the role of KIBS in regional and national innovation systems. In: Research Policy Bd. 30 (2001), S. 1501-1516.

[NOL 92] NOLLET J., HAYwOOD-FARMER J., Les entreprises de services. Gaëtan Mor, 1992.

[OEC 00] OECD: Promoting innovation and growth in services., 2000.

[OOEC 06] OECD: Innovation and knowledge intensive service activities, 2006.

[PAV 84] PAVITT K., Sectoral patterns of technical change: Towards a taxonomy and a theory. In: Research Policy Bd. 13 (1984), Nr. 1984, S. 343-373.

[ROM 90] ROMER P.M., Endogenous Technological Change. In: Journal of political Economy Bd. 98 (1990), Nr. 5, S. 71-102.

[SIM 06] SIMmIE J., STRAmBACH S., The contribution of KIBS to innovation in cities: An evolutionary and institutional perspective. In: Journal of Knowledge Management Bd. 10 (2006), Nr. 5, S. 26-40.

[SOE 89] SOETE L., Miozzo M., Trade and development in services : a technological perspective, 1989.

[TET 02] TETHER B.-S., HIPP C., Knowledge intensive, technical and other services: Patterns of competitiveness and innovation compared. In: Technology Analysis and Strategic Management Bd. 14 (2002), Nr. 2, S. 163-182.

[WER 18] Werner P., Strambach S., Policy mobilities, territorial knowledge dynamics and the role of KIBS: Exploring conceptual synergies of formerly discrete approaches. In: Geoforum Bd. 89, Elsevier (2018), Nr. December 2016, S. 19-28.

(c) 2021 ISTE OpenScience - Published by ISTE Ltd. London, UK - openscience.fr

Page $\mid 11$ 